\title{
A factor analytic study of hue discrimination
}

\author{
DON DIENER \\ University of Nevada, Las Vegas, Nevada
}

\begin{abstract}
Hue discrimination data were gathered from a variety of published sources and subjected to factor analysis. Five factors were extracted. Factor I affects discrimination performance in the orange-red portion of the spectrum, from 590 to $640 \mathrm{~nm}$ in wavelength. Similarly, Factor II contributes to performance in the violet-blue portion of the spectrum (430-490 nm), and Factor III does so in the middle wavelengths $(490-570 \mathrm{~nm}$ ). Factor IV affects performance in two separate portions of the spectrum, from 570 to $610 \mathrm{~nm}$ and from 450 to $490 \mathrm{~nm}$. Factor V affects performance from 470 to $530 \mathrm{~nm}$, with a peak loading at $510 \mathrm{~nm}$, the point of maximum absorption of rhodopsin. The implications of this factor pattern for theories of color vision are discussed, as is the application of factor analysis to psychophysical data.
\end{abstract}

Although factor analysis is widely used in personality research and test development, it is seldom employed in the traditionally "experimental" areas of psychology. There are several reasons for this restricted domain of application. Factor analysis is often dismissed as a "correlational method" which has little to offer in areas in which manipulative research is possible. The technique is usually represented even by its adherents as an "exploratory" procedure of little value in well-researched fields. Furthermore, there is a good deal of suspicion on the part of many researchers of both the data transformations employed and the process of interpreting the resulting factor patterns.

There are, however, reasons to recommend application of factor analysis to areas outside of its traditional domain. Like other correlational methods, factor analysis can make use of individual difference data. More traditional research methods often accord individual differences the same status as measurement error, discarding potentially useful information. Factor analysis also has the advantage of allowing the data, rather than the investigator, to suggest the theoretical entities used to summarize the data. Many of the problems associated with the use of factor analysis (e.g., lack of factor stability) may owe more to the fact that it has often been applied to data with a high degree of measurement error than to problems inherent in the technique itself. Thus, it seemed possible that the application of factor analysis to reliable (e.g., psychophysical) data could result in more highly stable and interpretable factors than those often found in personality research.

The most common form of factor analysis ( $R$ analysis) begins with a matrix of intercorrelations among a set of tests. This correlation matrix is then reduced to a smaller

I wish to thank Charles Burdsal, Wichita State University, Lori Kolbet, University of Nevada, Las Vegas, and an anonymous reviewer for helpful comments on earlier drafts of this paper. Requests for reprints should be addressed to: Don Diener, Department of Psychology, University of Nevada, Las Vegas, NV 89154. set of dimensions (factors). Interpretation of a factor analysis is usually based on inspection of the factor pattern, a matrix of weights which, when multiplied by subjects' factor scores, reproduce the subjects' (standardized) performance scores. Conceptually, the values of the factor pattern matrix ("factor loadings") represent the importance of a factor to a given test, whereas the factor scores represent the quantity of a particular factor possessed by an individual.

Under some conditions, factor analysis can reveal important characteristics of a system under study. Consider the application of factor analysis to a matrix of correlations among tests of hue discrimination at various wavelengths across the spectrum. Different factor patterns would be expected, depending on the nature of the mechanism underlying hue discrimination. Assume, for example, that differences in hue discrimination ability depend entirely on differences in the sensitivity of three Young-Helmholtz-type receptors with maximum excitability in the blue, green, and red portions of the spectrum, respectively. Assume further that the sensitivities of these receptors vary independently. Knowledge of the sensitivity of each of the receptors would then be required to reproduce a subject's hue discrimination curve. Under these conditions, a factor analysis should reveal three factors. The factor pattern would reflect the relative importance of each of the factors to discrimination at the various wavelengths-the peak loadings on the three factors should fall in the blue, green, and red portions of the spectrum. If, on the other hand, hue discrimination ultimately depended on information coded as the output of two opponent processes, two bipolar factors would be expected. One of the factors might be expected to have peak loadings in the yellow and blue portions of the spectrum and the other to have them in the red and green.

There are, however, limitations to such analyses. An $R$ analysis will reveal information only about processes that vary independently across subjects. If the three basic processes in a trichromatic system were perfectly correlated, only one factor could emerge. On the other hand, 
not all factors revealed by a factor analysis may be of theoretical importance. For example, under some conditions, an analysis of hue discrimination ability might reveal factors related to visual acuity.

There are also sources of indeterminacy in a classical factor solution, including estimation of communalities and factor rotation. The reader who is unfamiliar with approaches to these issues is referred to one of the many texts on the subject (e.g., Gorsuch, 1974; Harman, 1967). A number of criteria can be used to guide the process of rotation, most involving the ideas of parsimony ("simple structure") and interpretability. Different rotational schemes result in different factor patterns, sometimes suggesting different interpretations. Given the limitations and indeterminacy of factor-analytic solutions, the usefulness of factor analysis in the interpretation of psychophysical data should be regarded as an empirical matter.

Although there have been no recent examples of the application of $R$ analysis to psychophysical data, factor analysis has been used in the study of color vision in the past. Burt (1946) factored a data set, consisting of a variety of tests of color discrimination, using his "simple summation" method. He then rotated the resulting factors "to eliminate the negative saturations." The unrotated threefactor solution seemed compatible with Hering's opponent-process theory of color vision, whereas a rotated four-factor solution seemed more compatible with the Young-Helmholtz theory. Pickford (1951) used similar methodology to factor hue discrimination data for both normal subjects and several groups of subjects with a variety of color-vision deficits. Pickford found three factors; a general factor, a bipolar yellow-blue factor, and a bipolar red-green factor. The factor patterns of the various groups of color-blind subjects differed from those of the normal subjects and among each other. Pickford interpreted his results as supportive of the opponent-process theory of color vision.

Forms of factor analysis that do not depend on individual difference data have also been employed in the study of color vision. Ekman (1954) asked observers to provide similarity ratings for all possible pairs of 14 different hues. The matrix of mean similarity ratings was factored using Thurstone's group centroid method and rotated visually to simple structure. Five unipolar factors were extracted with peak loadings in the violet $(434 \mathrm{~nm})$, blue $(472 \mathrm{~nm})$, green $(537 \mathrm{~nm})$, yellow $(600 \mathrm{~nm})$, and red $(651 \mathrm{~nm})$ portions of the spectrum. Guth, Donley, and Marrocco (1969) employed similar methodology in a study of luminance additivity. These investigators measured the relative luminance of light at varying wavelengths necessary to bring a subthreshold field of a given wavelength to threshold. The cosines of the luminance-wavelength vectors relating pairs of stimuli (based on average values for all subjects) were subjected to a principal-components factor analysis. Three broadband bipolar factors were found. These factors were found to be consistent with a variety of color mixing data.
The type of factor analysis represented by the studies of Ekman (1954) and Guth, Donley, and Marrocco (1969) reveals information of a different kind from that revealed by traditional $\mathrm{R}$ analysis. The former analyses reduce the number of dimensions necessary to describe the average performance of a group of subjects rather than revealing the dimensions necessary to describe differences among subjects. Ekman's analysis, for example, revealed that subjects perceive colors as falling into five categories, best represented by wavelengths of $434,472,537,600$, and $651 \mathrm{~nm}$. Although this kind of information can be valuable, the R approach employed by Burt (1946) and Pickford (1951) seems more likely to reveal information about the basic processes underlying color vision. Unfortunately, the studies of Burt (1946) and Pickford (1951) suffered from a number of problems, including questionable data and the use of rather primitive factor-analytic techniques.

In the present study, modern factor analysis is applied to hue discrimination data collected from a variety of published sources. In addition to information that may be revealed about the process of hue discrimination, the application of factor analysis to a well-researched field such as color vision should be useful in assessing the potential value of applying the technique to domains in which less is known.

\section{METHOD}

\section{Data}

Hue discrimination data were collected from a number of published sources for a total of 24 subjects. Twelve subjects were taken from Stiendler (1906), 5 from Wright and Pitt $(1934,1935), 3$ from Laurens and Hamilton (1923), 2 from König (1903, cited by Wright, 1947), and 1 each from the studies of Jones (1917) and Nelson (1937). In all cases, data were in the form of the just noticeable difference in wavelength for each subject for standard stimuli at $20-\mathrm{nm}$ intervals from 430 to $630 \mathrm{~nm}$ and one test at $640 \mathrm{~nm}$. Data for 3 of the Wright and Pitt (1934) subjects, the 3 subjects of Laurens and Hamilton (1923), and the 2 König subjects were not available in tabular form and were estimated from the published graphs. Stiendler (1906) did not report data for all of her subjects at precisely the wavelengths used in the present study. However, data were reported within $2 \mathrm{~nm}$ of these wavelengths in virtually all cases. All values used in the analysis were rounded to two significant figures.

Different methods were used by the various authors in collecting the data. In many cases, it is not possible to determine the exact characteristics of the stimuli and procedures employed. Stiendler (1906) presented her subjects with a first-order spectrum $85 \mathrm{~cm}$ in length and obtained the minimum change in wavelength necessary to perceive a difference in hue between portions of a split field. The luminosity of the halves of the field was not equated for the subjects used in the present analysis. Average luminosity, although not specified, was, given the nature of the apparatus, probably quite low.

Wright and Pitt $(1934,1935)$ asked their observers to adjust the halves of a split field until they were just noticeably different in hue. The entire field subtended a visual angle of about $2^{\circ}$. The halves of the field were equated for luminosity, but different levels of intensity were used in different portions of the spectrum. For wavelengths between 480 and $650 \mathrm{~nm}$, the average retinal illumi- 
nation varied over a 4-to- 1 range with an average of 70 photons (trolands). As wavelengths decreased from 480 to $420 \mathrm{~nm}$, illumination was allowed to increase 20-fold. Nelson (1937) apparently used the same apparatus and procedures as Wright and Pitt.

Laurens and Hamilton (1923) asked their observers to report whether they could detect a change in hue as an assistant varied the wavelength of half of a split field ( $3^{\circ}$ of visual angle) in increasingly larger steps. The luminosity of the two halves of the field was equated, and constant brightness was maintained across the spectrum, but the level (4 photons) was rather low.

König's observers adjusted the halves of a visual field (size unspecified) until the hues appeared to match. The halves of the field were not equated for brightness, and the overall intensity level was not specified.

Jones (1917) adjusted the halves of a split field until they were just noticeably different. The luminosity of the portions of the field was equated. Neither the size of the field nor the overall intensity level was specified.

\section{Analysis}

The factor analysis was performed following the strictures of Cattell (1978) with respect to determining the number of factors, estimating communalities, and rotation to simple structure. A matrix of correlations among tests at various wavelengths was computed from the raw data. A principal-components analysis was applied to the matrix of intercorrelations. Eigenvalue plots from this analysis were used to determine the correct number of factors following the scree criterion (Cattell, 1966). Scree indicated five factors. This was confirmed by Bartlett's (1950) chi-square, which indicated no significant residual covariance after five factors were removed $\left[\chi^{2}(27)=23.5\right]$ but significant residual covariance with only four factors $\left[\chi^{2}(35)=58.8, \mathrm{p}<.01\right]$. A principal axis analysis using an iterative procedure to estimate communalities was then performed. Final communality estimates from this analysis ranged from .78 to 1.00 . A Varimax orthogonal rotation was applied to the five-factor matrix, followed by a series of visually guided oblique rotations and finally a Maxplane clean-up rotation, resulting in a $55 \% .10$ hyperplane count.

The data were also transformed in a number of ways and analyzed using different methods of rotation and different numbers of factors. The solution obtained by the above described procedure, however, was the most clearly interpretable and best on a number of measures. For those who might wish to test other solutions, a table of eigenvalues and eigenvectors appears in the Appendix.

\section{RESULTS AND DISCUSSION}

The factor pattern and factor correlations from the analysis are shown in Tables 1 and 2. Factor I contributes to discrimination performance in the orange-red portion of the spectrum, from 590-640 nm. Similarly, Factor II contributes to performance in the violet-blue portion of the spectrum (430-490 nm), and Factor III does so in the middle wavelengths (490-570 nm). Factor IV affects per-
Table 2

Factor Correlations

\begin{tabular}{cccccc} 
& \multicolumn{5}{c}{ Factors } \\
\cline { 2 - 6 } Factors & I & II & III & IV & V \\
\hline I & & & & & \\
II & .30 & & & & \\
III & .42 & .33 & & & \\
IV & .29 & .14 & .31 & & \\
V & .24 & .43 & .08 & .12 & \\
\hline
\end{tabular}

formance in two separate portions of the spectrum, from 570 to $610 \mathrm{~nm}$ and from 450 to $490 \mathrm{~nm}$. The pattern of loadings on Factor $\mathrm{V}$ is monotonic, like those of Factors I, II, and III, affecting discrimination performance from 470 to $530 \mathrm{~nm}$.

In order to assess the extent to which the various factors might depend on differences in the methods employed by the different investigators in the collection of the data rather than on differences among the subjects themselves, factor scores were computed from the orthogonal (Varimax) solution. Inspection of the factor scores revealed that all factors were represented in the data from each of the six sources included in the analysis. The scores for each of the five factors were subjected to separate analyses of variance comparing the groups of subjects from the six different data sources. The groups differed significantly in their scores on Factor $\operatorname{I}[\mathrm{F}(5,18)=5.86, \mathrm{p}<.01]$ and on Factor II $[F(5,18)=3.08, p<.05]$. The $F$ ratios for Factors III, IV, and V were smaller than unity.

Inspection of the mean factor scores for the groups revealed that the significant effect for Factor I was attributable to higher scores on the part of the subjects of König (cited by Wright, 1947) and Stiendler (1906). This was confirmed by Duncan's multiple range test and the Scheffé method ( $p<.05$ ). Unilike the other investigators, König and Stiendler did not equate the brightness of the halves of the split fields compared by their observers, possibly accounting for the superior performance of their subjects in the longer wavelengths. In the case of Factor II, the group differences appeared to be attributable to the higher factor scores of Wright and Pitt's (1934, 1935) observers. Duncan's multiple range test revealed that Wright and Pitt's subjects different significantly from the other groups $(p<.05)$. Wright and Pitt increased the illumination of the stimuli as wavelength decreased from 480 to $420 \mathrm{~nm}$. This increased illumination might be expected to result in superior performance on the short wavelengths

Table 1

First-Order Factor Pattern

\begin{tabular}{|c|c|c|c|c|c|c|c|c|c|c|c|c|}
\hline \multirow[b]{2}{*}{ Factor } & \multicolumn{2}{|c|}{ Violet } & \multicolumn{2}{|c|}{ Blue } & \multicolumn{2}{|c|}{ Green } & \multicolumn{2}{|c|}{ Yellow } & \multicolumn{2}{|c|}{ Orange } & \multicolumn{2}{|c|}{ Red } \\
\hline & 430 & 450 & 470 & 490 & 510 & 530 & 550 & 570 & 590 & 610 & 630 & 640 \\
\hline I & -.087 & .070 & .040 & .001 & .011 & .239 & .084 & -.049 & .407 & .728 & 1.008 & .875 \\
\hline II & 1.019 & .825 & .650 & .266 & -.014 & .071 & -.046 & .024 & -.041 & -.398 & -.113 & .092 \\
\hline III & -.122 & .100 & -.087 & .219 & .583 & .721 & .925 & .822 & .048 & -.021 & .029 & .017 \\
\hline IV & .012 & -.275 & .318 & .431 & .024 & .023 & -.055 & .400 & .720 & .527 & .034 & -.032 \\
\hline $\mathrm{V}$ & .023 & .021 & .303 & .432 & .710 & .221 & .073 & -.031 & .012 & .102 & -.129 & .031 \\
\hline
\end{tabular}


represented by Factor II. Despite these differences, none of the factors seem attributable to differences in the procedures employed in the data collection.

In examining the results of the factor analysis, it is necessary to consider the nature of the process of drawing inferences about underlying mechanisms from the factor pattern. The test at $630 \mathrm{~nm}$ has a salient loading only on Factor I. Because the factor pattern is derived from the matrix of intercorrelations among the tests, this single salient loading indicates that the covariability of the "scores" on the test at $630 \mathrm{~nm}$ can be almost entirely accounted for by the hypothetical structure represented by Factor I. Factor I also accounts for a substantial portion of the covariability in the other tests, from 590 to $640 \mathrm{~nm}$. Thus, Factor I represents the tendency of the tests in the red portion of the spectrum to covary. The higher the positive loading of a test at a given wavelength on this factor, the more predictive or representative is performance at this wavelength of performance on tests at other wavelengths with high loadings on Factor I. A negative loading of a test on Factor I would indicate a tendency for performance at the wavelength on which the test is based to vary inversely with performance on the tests with positive loadings.

The pattern of loadings on Factors I, II, and III suggests that these factors are based rather directly on the action of the three types of cones. Although the peak loadings on these factors at 430,550 , and $640 \mathrm{~nm}$ do not fall precisely at the points of peak absorption of primate cone pigments (i.e., 445, 535, and $570 \mathrm{~nm}$; Marks, Dobelle, \& MacNichol, 1964), they fall in portions of the spectrum in which the ratio of the absorption of the primary pigment to the others is greatest. The test with the highest loading on Factor $\mathrm{V}$ falls at $510 \mathrm{~nm}$, which corresponds to the point of peak absorption of rhodopsin (Rushton, 1962). Factor V would, therefore, seem to be related to the activity of the rods.

The pattern of Factor IV with peaks in the blue-green and yellow portions of the spectrum is more difficult to interpret. An obvious possibility is that Factor IV is based on a Hering-type (opponent-process) mechanism. However, the rest of the factor pattern (e.g., the lack of a comparable red-green factor) does not fit well with this interpretation.

It is possible to speculate further about the nature of the mechanisms underlying the various factors by reference to the mean performance on the original discrimination task shown in Figure 1. There are several wavelengths at which, according to the present analysis, performance is based almost entirely on Factors I, II, or III, that is, wavelengths with salient loadings only on one of these factors. The tests at 630 and $640 \mathrm{~nm}$ have salient

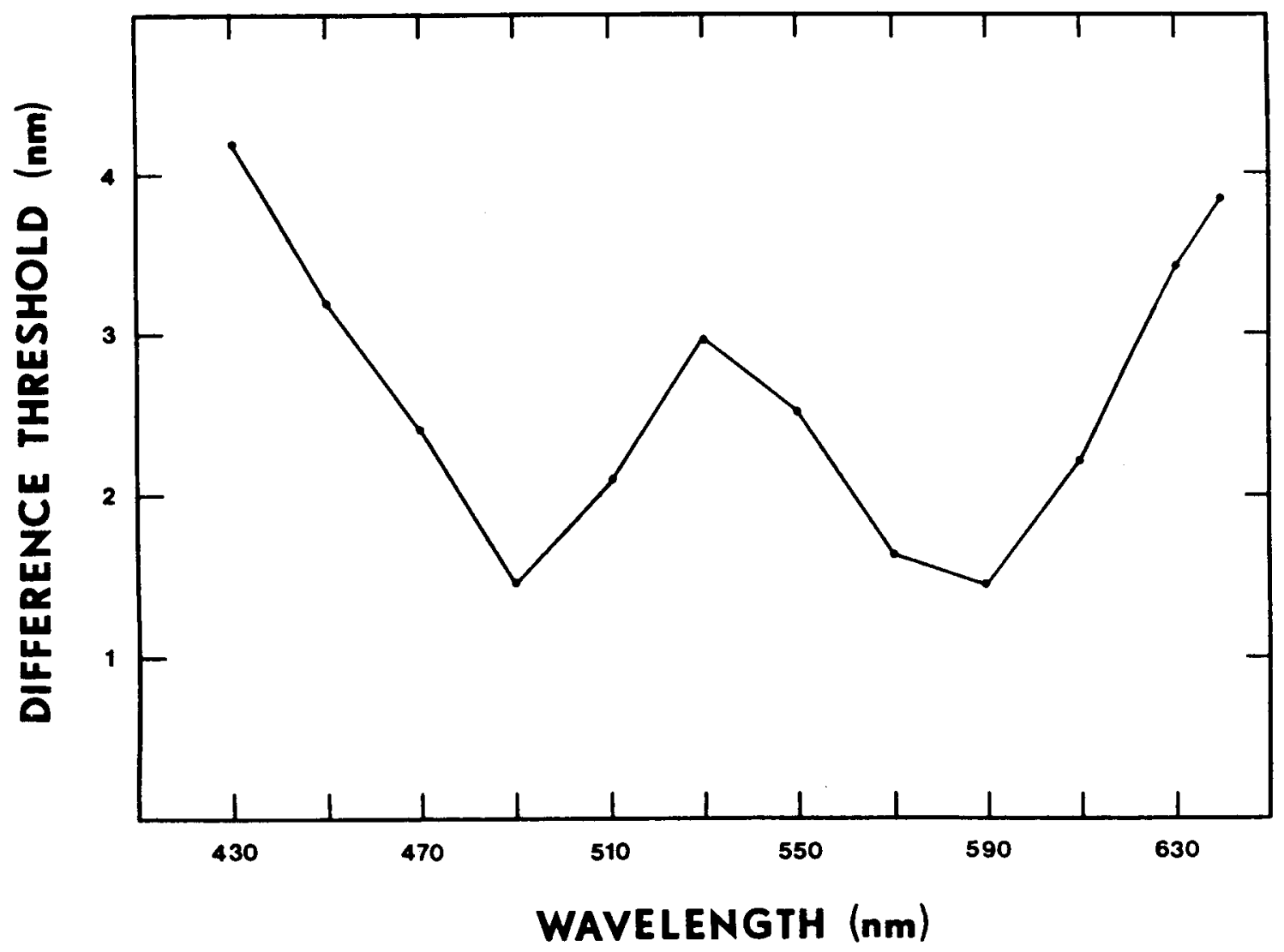

Figure 1. Average difference threshold as a function of wavelength for the 24 subjects. 
loadings only on Factor I, those at 430 and $450 \mathrm{~nm}$ have salient positive loadings only on Factor II, and the test at $550 \mathrm{~nm}$ has a salient loading only on Factor III. These tests fall in portions of the spectrum in which hue discrimination is relatively poor. The two peak loadings on Factor IV at 490 and $590 \mathrm{~nm}$, on the other hand, fall precisely at the two local minima in the hue discrimination curve. The mechanisms underlying Factor IV would, therefore, seem to be more sensitive than those underlying the first three factors. Due to the considerable overlap of the pattern of Factor V with the other four factors, it is not possible to draw strong conclusions about the relative sensitivity of the underlying mechanism.

The fact that hue discriminability is relatively poor in the portions of the spectrum corresponding to the peak loadings on Factors I, II, and III is compatible with the interpretation that these factors are based on the activity of single receptors. That is, that discrimination of small differences in wavelength in the approximate ranges of 430 to $460 \mathrm{~nm}, 520$ to $560 \mathrm{~nm}$, and 610 to $640 \mathrm{~nm}$ is based on changes in the degree of excitation of the single receptor most sensitive at these wavelengths. Discrimination based on the degree of excitation of a single receptor should be poorer than discrimination based, for example, on a mechanism that compares the degree of excitation of pairs of receptors. This latter mechanism might be the basis for Factor IV, which has peak loadings at the local minima of the hue discrimination curve. According to this interpretation, the loadings of the tests at 570-610 nm on Factor IV represent discrimination based on the relative excitation of the green- and red-sensitive cones, whereas the loadings of the tests at 470 and $490 \mathrm{~nm}$ represent discrimination based on the relative excitation of the violet- and green-sensitive receptors.

It seems worthy of note that the tests with peak loadings on the present five factors correspond almost perfectly to the wavelengths producing maximum activity in cells of the lateral geniculate nucleus (LGN) identified by DeValois (1960). DeValois identified five different populations of cells in the LGN of primates ("narrow band 'on' cells') which were maximally responsive to stimulation by light at $430,510,550,590$, and $630 \mathrm{~nm}$, respectively. Although DeValois later changed his interpretation of these data (DeValois, Jacobs, \& Jones, 1962), they suggest a possible physiological mechanism underlying the factors.

Several minor features of the factor pattern are also interpretable. The -.275 loading of the test at $450 \mathrm{~nm}$ on Factor IV suggests that the more an observer relies on the mechanism underlying Factor IV, the worse performance will be at $450 \mathrm{~nm}$. A possible explanation for this loading is that the mechanism underlying Factor II provides more accurate information about hue differences around $450 \mathrm{~nm}$ than does Factor IV. A tendency to rely on Factor IV would therefore result in relatively poorer performance at $450 \mathrm{~nm}$.

The .239 loading of the test at $530 \mathrm{~nm}$ on Factor I and the negative loadings of the tests at 610 and $630 \mathrm{~nm}$ on Factor II indicate a slight tendency for performance on tests in the red and green portions of the spectrum to vary directly and for tests in the blue and red portions of the spectrum to vary inversely. This pattern of covariation might be attributable to differential spatial distribution of the receptors associated with these factors on the retina (i.e., foveal vs. parafoveal). If this interpretation is correct, however, we would expect to find similar small positive loadings of the tests in the red portion of the spectrum on Factor III. A possible explanation for the absence of these loadings is that the foveal-parafoveal effect is partially represented in the factor pattern and partially represented in the correlation among the factors. A simple second-order analysis was conducted to investigate this possibility. The factor correlation matrix was subjected to a principal-component analysis, yielding two factors with eigenvalues greater than 1 . The resulting factor pattern appears in Table 3.

Second-order Factor I is a general factor representing the tendency for all of the first-order factors (performance on all of the tests) to vary directly. Second-order Factor II represents the tendency for first-order Factors II and $\mathrm{V}$ to vary directly and to vary inversely with first-order Factors I, III, and IV, that is, for the accuracy of discrimination judgments involving parafoveal receptors to vary inversely with the accuracy of judgments involving foveal receptors. A number of factors could contribute to this relationship. Both characteristics of the individual subjects (e.g., "nearsightedness"), and characteristics of the procedures employed by the various experimenters who collected the original data (e.g., stimulus size and distance from the subject), may have influenced the spatial distribution of light on the observer's retina. The higher the degree of foveal focus of the light, the better the performance on the tests involving the red- and greensensitive receptors (Factors I and III), but the worse the performance on tests based on Factors II and V. Although Factor IV probably involves both foveal and parafoveal receptors, it accounts for a larger proportion of the variance in the tests from 570 to $610 \mathrm{~nm}$ than in the range of 450 to $490 \mathrm{~nm}$ (witness larger factor loadings). Factor IV is therefore grouped with first-order Factors I and III on second-order Factor II, rather than with Factors II and V.

\section{THEORETICAL IMPLICATIONS}

Any successful theory of color vision must account for the shape of the hue discrimination curve shown in Figure 1. Theorists have attempted to derive this hue dis-

Table 3

Second-Order Factor Pattern

\begin{tabular}{crr}
\hline $\begin{array}{c}\text { First Order } \\
\text { Factor }\end{array}$ & \multicolumn{2}{c}{ Second-Order Factor } \\
\cline { 2 - 3 } I & II \\
\hline I & .728 & -.189 \\
II & .698 & .439 \\
III & .689 & -.400 \\
IV & .540 & -.485 \\
V & .547 & .675 \\
\hline
\end{tabular}


crimination function from a number of theoretical perspectives (e.g., Hecht, 1934; Helmholtz, 1896, cited in Peddie, 1922; Hurvich \& Jameson, 1955; Stiles, 1946). All of these theorists started with a set of assumptions compatible with their theoretical orientation and then made additional assumptions necessary to derive the hue discrimination function. In all cases, the fit of the theoretical curves to the data was satisfactory. The status of the various theories has risen or fallen, not on the basis of their ability to account for the hue discrimination curve, but rather on an evaluation of the assumptions necessary to derive the curves.

Consider the earliest of these formulations as an example. Helmholtz (1896, cited in Peddie, 1922) based his explanation of hue discrimination performance on changes in the intensities of three fundamental response processes maximally sensitive at short (S), middle (M), and long (L) wavelengths. For a given wavelength the just noticeable change in wavelength $(\Delta W \lambda)$ is related to changes in the fundamental response processes by:

$$
\Delta \mathrm{W} \lambda^{2}=\Delta S \lambda^{2}+\Delta \mathrm{M} \lambda^{2}+\Delta \mathrm{L} \lambda^{2} .
$$

The quadratic nature of the formula is imposed to constrain the value of $\Delta W \lambda^{2}$ such that $W \lambda^{2} \geq 0$ and $\Delta W \lambda^{2}=0$ only if $\Delta S \lambda^{2}=\Delta M \lambda^{2}=\Delta L \lambda^{2}=0$. Hue discriminability in a given portion of the spectrum is dependent upon the degree of change in the underlying response processes associated with a change in wavelength. At those points on the spectrum where two or three of the response processes change rapidly, discriminability is good. On the other hand, in the portions of the spectrum where a change in wavelength causes a change in only one of the response processes, at very long wavelengths for example, discriminability is poor.

Helmholtz derived fundamental response functions which would produce the observed hue discrimination function in accordance with Fechner's law. Although these fundamental functions gave an adequate fit to the hue discrimination curve, they have been objected to on several grounds. Stiles (1946) lists three objections. First, each of the response functions has a two-peaked response spectrum. Second, the form of Equation 1 does not follow $\mathrm{Ab}$ ney's additivity law. Third, the luminosity function which would result from Helmholtz's values has its peak at too low a wavelength and is generally too high in the blue portion of the spectrum. Although these objections may not be as serious as once thought (see Wasserman, 1977, for a discussion), they were sufficient to cause most theorists to abandon Helmholtz's position. The theoretical formulations which succeeded Helmholtz's followed similar patterns with respect to formulation and evaluation.

Rather than beginning with a set of theoretical entities and attempting to synthesize the hue discrimination curve, the factor analytic approach employed in the present study is an attempt to derive theoretical entities (factors) from the individual hue discrimination curves. Under most sets of assumptions, we would expect to find similarities be- tween the factor pattern and the "true" function relating hue discrimination performance to the basic underlying processes. Assume for example that Helmholtz's position is correct and that (1) observers can differ with respect to the sensitivity of any of the basic response processes, and (2) these differences are at least partially independent of each other. Under these assumptions, we would expect to find three factors corresponding to $S, M$, and $\mathrm{L}$ wavelengths. The factor pattern would be expected to reflect the importance of each of the basic response processes to discrimination at the various wavelengths. Because Helmholtz postulated two-peaked spectra for each of the response processes, we would expect to see two peaks in the loadings on each of the factors. However, this is not essential to the basic trichromatic position.

The findings of the present study are compatible with a modified trichromatic theory. Factors I, II, and III can be interpreted as representing the variation of subjects in the sensitivity of the basic response processes. Factor IV can be understood as representing variability in the observers' ability to base discrimination judgments on changes in the relative excitation of two receptors. Factor $\mathrm{V}$, which has been interpreted as representing varying sensitivity of the rods, represents a departure from trichromatic theory. Most discussions of color vision begin by ruling out any role of the rods as color receptors, on the basis of observations such as the fact that animals that have only these structures do not discriminate color and the fact that the color sensitivity in humans is lost when luminosity is reduced to the point at which only the rods are sensitive. Clearly, hue discrimination is impossible for a monochromatic system. However, because the rods have response spectra that differ from those of the other receptors, they should provide information about wavelength in a "tetrachromatic" system.

The present findings are difficult to reconcile with an opponent-process explanation of hue discrimination. Opponent-process theory would predict covariability among tests in the red and green portions of the spectrum as well as among tests in the yellow and blue. Thus, we would expect to find two factors similar to the present Factor IV, one with peaks in the red and green and the other with peaks in the yellow and blue. An adherent of this position might argue that Factors IV and V are imperfect representations of these opponent processes. Factor IV is clearly amenable to this interpretation. The interpretation of Factors I, II, and III, however, poses a serious problem for the opponent-process position. If hue discrimination ultimately depends on two opponent processes, variation in the sensitivity of the receptors should be represented only in their contribution to the opponent processes. That is, an observer's discrimination performance should be predictable by only two factors, corresponding to the opponent processes.

Althought the present analysis cannot be taken as conclusive, it provides evidence supportive of the trichromatic view of hue discrimination and suggests avenues for further research. Especially notable in this regard is the evi- 
dence of involvement of the rods in color vision, represented by Factor V. Given the problems attendant to the interpretation of data drawn from subjects tested under different (and in some cases unknown) conditions, it might be worthwhile to analyze hue discrimination data from a larger sample of subjects collected under uniform conditions.

The interpretability of the present analysis seems sufficient to suggest that factor analysis might be profitably employed in the examination of other psychophysical data. The technique is particularly suited to cases in which response functions along a single dimension (such as wavelength or intensity) are thought to result from the action of a number of distinct and potentially independent processes.

\section{REFERENCES}

BARTLETT, M. S. (1950). Tests of significance in factor analysis. British Journal of Psychology, 3, 77-85.

BURT, C. (1946). The relationship between eye color and defective color vision. Eugenics Review, 37, 149-156.

Cattell, R. B. (1966). The scree test for the number of factors. Multivariate Behavioral Research, 1, 245-276.

Cattell, R. B. (1978). The scientific use of factor analysis. New York: Plenum.

DEVALOIS, R. L. (1960): Color mechanisms in the monkey. Journal of General Physiology, 43, 115-128.

DeValois, R. L., JACOBS, G. H., \& Jones, A. E. (1962). Effects of increments and decrements of light on neural discharge rate. Science, 136, 986-987.

Eкman, G. (1954). Dimensions of color vision. Journal of Psychology, 38, 467-474.

Gorsuch, R. L. (1974). Factor analysis. Philadelphia: Saunders.

Guth, S. L., Donley, N. J., \& Marrocco, R. T. (1969). On luminance additivity and related topics. Vision Research, 9, 537-575.
HaRMAN, H. H. (1967). Modern factor analysis. Chicago: University of Chicago Press.

HECHT, S. (1934). Vision II. The nature of the photoreceptor process. In C. Murchison (Ed.), Handbook of general experimental psychology (pp. 704-828). Worcester, MA: Clark University Press.

Hurvich, L. M., \& JAMESON, D. (1955). Some quantitative aspects of an opponent-colors theory. II. Brightness, saturation, and hue in normal and dichromatic vision. Journal of the Optical Society of America, 45, 602-616

JoNES, L. A. (1917). The fundamental scale of pure hue and retinal sensibility to hue differences. Journal of the Optical Society of America, 1, 63-77.

Laurens, H., \& Hamilton, W. F. (1923). The sensibility of the eye to differences in wave length. American Journal of Physiology, 65, 547-568.

Marks, W. B., Dobelle, W. H., \& MacNichol, E. F. (1964). Visual pigments of single primate cones. Science, 143, 1181-1183.

NELSON, F. H. (1937). The color-vision characteristics of a trichromat, part 2. Proceedings of the Physical Society of London, 49 , 332-337.

Peddie, W. (1922). Colour vision. London: Edward Amold.

Pickford, R. W. (1951). Individual differences in color vision. New York: Macmillan.

Rushton, W. A. H. (1962). Visual pigments in man. Scientific American, 207, 120-132.

Stiendler, O. (1906). Die Farbenempfindlichkeit des normalen und farbenblinden Auges. Sitzungsberichte Akademie MathematicNaturwissenschafien, Wien Ila, 115, 39-62.

StILES, W. S. (1946). A modified Helmholtz line-element in brightnesscolor space. Proceedings of the Physical Society of London, 58, 41-64.

Wasserman, G. S. (1977). Color vision: An historical introduction. New York: Wiley.

WRIGHT, W. D. (1947). Researches on normal and defective color vision. St. Louis, MO: Mosby.

WRIGHT, W. D., \& PITT, F. H. G. (1934). Hue discrimination in normal color vision. Proceedings of the Physical Society of London, 46, 459-473.

Wright, W. D., \& PITT, F. H. G. (1935). The colour-vision characteristics of two trichromats. Proceedings of the Physical Society of London, 47, 205-217.

Appendix

Eigenvalues and Eigenvectors for the First Five Factors

\begin{tabular}{|c|c|c|c|c|c|}
\hline \multirow{2}{*}{$\begin{array}{l}\text { Wavelengths } \\
\text { (nm) }\end{array}$} & \multicolumn{5}{|c|}{ Factors } \\
\hline & I & II & III & IV & $\mathrm{V}$ \\
\hline 430 & .47694 & .77826 & .27635 & -.00663 & .18844 \\
\hline 450 & .49810 & .69881 & .20952 & .34285 & .10931 \\
\hline 470 & .69810 & .50140 & .26785 & -.31256 & -.00344 \\
\hline 490 & .79415 & .23407 & -.04061 & -.42105 & -.13600 \\
\hline 510 & .79624 & .21544 & -.26498 & -.04651 & -.44936 \\
\hline 530 & .89499 & -.00609 & -.27897 & .21393 & -.02997 \\
\hline 550 & .74655 & -.07704 & -.52218 & .31742 & .08513 \\
\hline 570 & .80227 & -.14646 & -.47523 & -.03887 & .27758 \\
\hline 590 & .73895 & -.41315 & .12848 & -.39266 & .24352 \\
\hline 610 & .63701 & -.67394 & .22295 & -.20848 & -.03950 \\
\hline 630 & .62620 & -.50826 & .44030 & .33515 & .00957 \\
\hline \multirow[t]{2}{*}{640} & .74410 & -.25653 & .44285 & .31752 & -.12948 \\
\hline & 6.12028 & 2.42307 & 1.29640 & .95355 & .43080 \\
\hline
\end{tabular}

(Manuscript received August 6, 1984; revision accepted for publication October 14, 1985.) 